\title{
Machi Zugu: Ser Machi
}

José Quidel (1).

"Iñiy am ta machigean pikefuy, müna kuxankawüngey ta machigen, fentepun füxa zugu ta $t i)(2)$

El presente trabajo tiene como primer objetivo provocar una discusión interna acerca de la institución del machi. Por ende un objetivo formativo hacia nuestra sociedad como asímismo hacia la juventud que acude al respaldo escrito de los conocimientos mapuche.

El segundo objetivo es más bien de carácter político cultural dirigido a la sociedad nacional que aparece necesario ante las reiteradas imprecisiones que ocurren permanentemente acerca del tema de lo que es SER machi, MACHIGEN; específicamente a las definiciones de machi que surgieron en un texto escolár de circulación nacional y el cual provocó un alto nivel de crítica en las distintas esferas de las instituciones mapuche y no mapuche.

Por tales motivos nos hemos propuesto la tarea de lograr un acercamiento al concepto, al rol desde una perspectiva propia, desde adentro, desde lo mapuche.

Para ello, a modo de metodología, hemos recurrido a los propios actores que nos digan, nos hablen de su realidad, de su especialidad, del oficio de ser machi en el pueblo mapuche. Asimismo hemos estado participando de sus ceremonias y trabajo propio de su oficio.

Con ello queremos ir adoptando como metodología la sabiduría mapuche que versa sobre el hablar con fundamento, re pipigekelay ta che, müley ta ñi küme zugual ta che, rüf zugual. Lo cual permite una construcción responsable del discurso.

En este espacio queremos hacer valer el principio de hablar de lo nuestro. No pretendemos introducirnos en disciplina alguna para lograr tal objetivo. Nuestra experiencia, pertenencia y praxis en nuestra sociedad, creemos que es más que suficiente para hablar de lo nuestro. Desde luego valoramos altamente la posibilidad de un espacio en el cual podamos difundir nuestras propias cosas.

Otra de las razones que nos impulsa a escribir, es más bien la de otorgar una orientación y algunos elementos claves que contribuirán a un trabajo cada vez más acertado sobre la temática mapuche, como a la de contribuir a una relación interétnica e intercultural más equitativa, responsable y respetuosa.

Esperamos que este trabajo sea uno de los primeros artículos que intentarán modestamente seguir profundizando acerca de los diversos aspectos de nuestra sociedad.

De antemano agradecemos la confianza y la disponibildad de mis hermanas y hermano machi, que me dieron parte su valioso tiempo para concretizar mi trabajo. Chaf mapuche am iñchin,, müley jemay ta iñ xür küzawküleal fijke zugu mew. Wenuntunierpuayiñ ta iñ mapun rakizuam, kimelafiyiñ ta iñ pu che ka ti pu kake xipa che.

\section{TA IN MAPUNCHE RAKIZUAM \\ ALGUNOS ALCANCES ACERCA DE NUESTRO PENSAMTENTO MAPUNCHE}

El hombre ocidental habla de lo natural como lo tangible y cataloga como sobrenatural aquello que no calza en esta categoría. Para nosotros es el mundo de los newen. Fuerzas que coexisten con nosotros, y dan sustento a la vida natural. Ello significa que cada manifestación de la naturaleza es una fuerza. El kürüf, viento; aht'ü, sol; mawüh, la lluvia; küyeh, la luna; xalkan el trueno y cada una de ella son fuerzas vitales, pues contribuyen a formar el todo, en el naüq mapu. Si una de las fuerzas deja de existir, la naturaleza entra en un

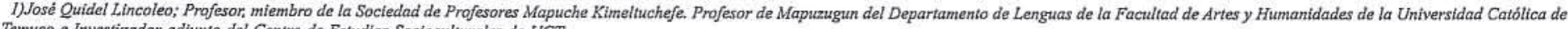
Temuco e Investigador adjunto del Centro de Estudios Socioculturales de UCT. 
desiquilibrio y acontecen hechos o repercusiones que catalogamos generalmente de negativos. Cada época del xipantu, (año) es también un newen. En el walüg, (verano) tendremos el newen del aht'ü, en el pukem (invierno) el newen del mawüh, en el rimü (otoño) el newen del kürüf, y en el pewü o (primavera) el newen del choyin, jeqün y el rayin.

A la vez es bueno que en cada época acontezca lo esperado.

«Porque cada cosa es funcional para si mismo, el agua es funcional para ella misma, las plantas por ejemplo, el desarrollo de la planta fortalece su propia fuerza independientemente que ayude la otra, ello contribuye a la totalidad. El hombre es nada sin la naturaleza, si pierde todo no podría ser hombre no podría SER. De ahi viene toda la definición, hablar de mawiza, anümka, que son infinidades de nombres y usos que tienen. Situémosno en el caso, si todos nos transformáramos en árbol, serviríamos para cada cosa en la cuál nos podrían usar». Victor Caniullan.(3)

Cuando hablamos de püjü, nos referimos al suelo por un lado y a lo que en castellano se denomina espíritu. En la concepción mapuche el término püjü denota lo material, lo tangible y lo etéreo. Pero en la filosofía mapuche ambas entidades se asemejan y en un momento de la «vida» se conjugan. En este contexto entendemos la vida tanto terrenal como ultraterrenal.

Los mapuches no adoramos dioses. Nos relacionamos con fuerzas que coexisten con nosotros en la naturaleza y nos dan sustento. Günenmapun, un ser que no reconoce al hombre como centro del mundo, sino sólo como parte de un cosmos más integral, el mapu. El mapu es el sustento del che y el mapu está conformado por los newen. El che una vez cumplido su ciclo vital se transformará también en mapu, y dará sustento a nuevas vidas.

Cada ecosistema posee un newen. Los geh o dueño de los ecositemas. En este contexto existe un Güenenmpun. El lof en donde existe y $\mathbf{E S}$ el che, existe el günenmapun, y cohabitamos con él y hacia él es con quién realizamos nuestros ritos, ello porque, nuestro sustento está en el mapu. Vivimos en ella, del püjü como suelo y con un püjü espíritu, nuestro newen.

El hombre es por esencia una parte más de un complejo y completo sistema interrelacionado como lo es la naturaleza toda.

\section{KIÑEKE ÜY AZ}

NOMBRE DE ALGUNOS ELEMENTOS, SUS FUNCTONES Y SIGNIFICADO.

«El rewe se compone de diversos vegetales como el maqui, el canelo, la quila, el manzano y el helecho ampe. En medio de estas ramas se coloca un palo con peldaños (el rehue estrictamente dicho) para que en él se extasíen las machis». Coña (1995).

El rewe es el símbolo exclusivo de la institución del machigen. Está compuesto de un madero nativo con algún número de peldaños que sirven para que la machi ascienda en su küymin Además, en los peldaños reposan los elementos para el pewütun.

Este madero transcurrido unos años se deteriora por lo cual debe ser renovado. Esta renovación consis- . te en una ceremonia de carácter religioso-social que recibe el nombre de geykurewen.

Pero el rewe también posee una segunda connotación muy relevante. Es lo referente a la organización social-religiosa que la conforman varios lof mapu, y se plasma en el gijatuwe. Estoy refiriéndome principalmente a la estructura wenteche.

Kulxug, es el nombre común con el cual se le conoce al instrumento principal de los machi, el cual cumple diversas funciones. En el lenguaje ritual, este instrumento adopta el nombre de kawiñkura. Este instrumento en algunos rituales se acompaña con la kaskawija, instrumento de origen occidental consistente en borrlas metálicas, generalmente de bronce.

Pero el kulxug es mucho más. Según el machi Caniullan, en el kulxug está el conocimiento, la educa- 
ción y el pensamiento mapuche. Es un instrumento que contiene sabiduría, en ella está la medicina, la religión, la astronomía, la representación del universo visto desde lo mapuche.

Junto al rewe se instalan las fantera, bandera de colores azul y blanco generalmênte, las que simbolizan el azul del cielo kajfui. Debemos recordar que el kajfü es el color representativo de los günemapun y de los componentes de la gran familia que nos ha creado, ta iñ pu reyñma iñ jeüqetew, kajfü wenu fücha, kajfü wenu kushe, kajfü wenu weche wenxu, kajfü wenu üjcha zomo. Por otro lado el liüq simboliza a las nubes en tiempo de walüg.

\section{$\measuredangle$ ¿HEM TA MACHI?}

¿QUE ES EL O LA MACHX?

Machi ta che. Elkunugey ta mapu mew ñi kejual $\tilde{n} i$ pu che kuxankawkülele, ñi gijatual, ñi ulal xemon zugu ñi pu kuxankawkülelu mew.

Machi ta lukutupelu, wül gübampelu, wül rakizuampelu.

El autor mapuche Martin Alonqueo por su parte especifica las diferencias de los términos machin de machi, con respecto al machin argumenta: «es una institución orgánica socio- religiosa que hay dentro del pueblo mapuche, cuya finalidad es ejercer una función netamente humanitaria que involucra ideas de fraternidad, de amor, que encarna un profundo idealismo espiritual alimentado por el espíritu de Dios (Ffil.eú), que significa la llama encendida del amor espiritual, del poder grande, de la fortaleza y la llama poderosa de amory temor».

Refiriéndose específicamente a la machi señala: «Es el título que ostenta la persona dedicada a esta profesión y es miembro activo de la institución»

Nøsotros señalaremos que antes que nada la o el machi es una persona que ha sido destinada a servir a sus iguales en el naüq mapu. Es la persona elegida que ayudará a mitigar el dolor de las personas, será la persona que guíe las rogativas, que levante las ceremonias y se arrodille en la tierra para pedir por sus hermanos: Otorgar sanidad a los enfermos, es conocedor de los baweh (medicina) que brotan, crecen, y se desarrollan en el püjü.

La machi es la persona a quien se le entrega el kimün a tavés del küymin y el gübam para que ella lo haga saber a sus semejantes en el naüq mapu, en el püjü mapu.

Fey mew ta elgey ta machi, machi ta pekan elgelay, iñiy no rume iñche ta machigean, iñche ta kimün ta zugu, iñche ta kimün ta güxam, iñche ta kimün ta rakizuam, iñche ta rumeñma azümniefin tüfa chi zugu, fey mew ta machigean ta. iñche pikelay iñiy no rume, iñiy no rume ayilay ñi machigeal, iñche afkentu kuxankawün ñi machigeal, eymün chi güxamkakefiymün chi machi, welu ixokom machi afkentu kuxankawüy, afkentu rupay ta güman mew, fey wüla wechupukey ñi zugu, wechupukey ñi güxam. Machi müley ñi niael, ñ $i$ elugeael gübam, fey ta müley, re üwüh mu liftu rukaal müten mülelay, ixokom küzaw mew müley ta ñi miawal machi. Ixokom rakizuam ñi nornial, gübamnial ñ $i$ guluche, fey mew ta elgey ta machi. Fey mew ta kimlu mapuche rakizuam, kimlu mapuche gübam, kimnieygün ka femgechi ka chumgechi elgeken machi. Wülniekey ta gübam, famgechi amuaymün, famgechi müleaymün, norümaymün zugu pilekey Caniullan.

Por ello ha sido creado la machi, la machi no ha sido creado por una mera casualidad o accidente, pues nadie puede atribuirse este rol y decir yo sere machi, yo sé, yo manejo los discursos y la conversación con sus respectivas pautas culturales formales, yo poseo el conocimiento, yo conozco el origen y el desarrollo del conocimiento mapuche en su profundidad, o decir; yo manejo y soy capaz de manejar esta institución, por ello sere machi, ello nadie puede osar decirlo. Pues a nadie le gusta tener que asumir el ser machi en el comienzo.

Yo sufrí mucho para llegar a ser machi, quizás ustedes han conversado con alguna machi, pero todos los machi hantenido que sufrir mucho para llegar a serlo, han tenido que derramar muchas lágrimas, recien entonces se completa su formación y los componentes del conocimiento que deben emanar de los discursos formales del mapuzugun.

El machi debe poseer, y de hecho se le dota de consejos, principios y normas para intervenir en todo trabajo mapuche, no sólo para exorcisar casas o funcio- 
nes únicas que generalmente se lo atribuyen las personas comunes. Debe de normar todo accionar mapuche, todo pensamiento mapuche, aconsejar, guiar, a sus semejantes. Por ello todo mapuche que conoce su pensamiento su filosofia, y los principios mapuches, conocen también el como llegan a formarse los machi.

Todos los machi son distintos, pues están relacionados con distintas fuerzas. Las fuerzas son propias de los elementos de la naturaleza.

Respecto del género, los machi pueden ser mujeres u hombres. En la antigüedad, la mayoría coinciden en que habían más machi hombre, pero luego esta institución se fue volcando más hacia las mujeres, lo cual no significa que no haya machi hombres en la actualidad.

Consejera, orientadora del mapuche rakizuam. Ella es conocedora y ejecutora permanente del mapun rakizuam. Por ello dirige, controla, sanciona, evalúa, y reorienta las conductas de los habitantes de su lof y de sus pacientes. Portadora de los profundos elementos culturales de su pueblo.

Nosotros podemos afirmar que la machi es considerada o considerado un especialista. Es una persona que por descender de un tronco familiar determinado ha tenido que asumir un rol pre-establecido por sus antepasados y llegado el momento la naturaleza se ha apoderado de él, para hacer que asuma su destino y su rol en el mapu.

Todo machi posee lo que podríamos denominar su código de ética. El cual por medio del gübam o el pewma recibe durante su período de formación que le reáliza su maestro (a) ta ñi machiletew. Además a través del pewma, sueño, y del küymin a la machi se le va corrigiendo, su actuar, se le va orientando y prediciendo los acontecimientos a su alrededor.

\section{Algunos principios que orientan el rol de machi.}

Chew rume wichagepalmi müley mi amual,

La no negación a su oficio, el tener que acudir donde sea requerida, cualquiera sea su condición.
Müley mi chofünuafiel che, ñagkolechi che müley mi fürenieafel mi kimün mew.

A ninguna persona se le ha de negar atención alguna, no debe de existir la negligencia en el espíritu de la machi.

Wifentu mapukey ta machi ñi küzaw mew.

A todas partes, en todos los rincones de la tierra, acudirá si así lo estiman los hermanos que necesitan su ayuda.

\section{Algunas razones por las cuales los padres o los llamados a Ser machi, no aceptan.}

\section{Kuxankawünkechi machigekey ta che.}

El hacerce machi implica en primer lugar un profundo sacrificio de la persona elegida, por lo cual ningún padre está dispuesto a ver que sus hijos padezcan de ello.

Ragi wühgekey ta machi ka fij zugu wixamelgekey ti bawehgelu; kalku, weza machi.

Estyarás en boca de todos, por tus actos, muchas veces has de ser incomprendido.

No todos entienden lo que significa ser machi, por lo tanto emiten juicios o más bien poseen prejuicios a priori los cuales muchas veces dañan a la persona como a la institución a la que pertenece. Es acusada de bruja, de tranzar con el mal, de manejar el mal, de ser mentirosa, que es un acto pagano, o de adoración al demonio, esto se denomina zuguyewün. Existen casos en que gran parte de la familia de la persona llamada a ser machi se ha opuesto terminantemente. La razón fundamental, es que la familia posee inclinaciones religiosas no mapuche. Generalmente estos grupos califican a toda manifestación religiosa mapuche e incluso manifestacipones sociales como algo pagano, primitivo, incluso demoníaco o diabólico.

En el tema del machigen, hemos conocido lamentables casos en que la propia persona llamada a asumir el rol, no accede, por sus creencias. La consecuencia es muy dramática, ella sufre de graves dolores físicos producto de la deformación de su estructura ósea, e incluso en época de pewü cuando brotan las yerbas medici- 
nales, choyün mew ta baweh, la persona sufre graves desiquilibrios psíquicos. Lo único a que la familia atina, es a internarla en el siquiátrico de la ciudad, cuando no funciona el ungimiento en la iglesia, las consecuencias para la persona son altamente nocivas.

Conocemos otros casos muy de cerca que bien podríamos calificarlos como agresión religiosa. Personas mapuche que arrancan rewe y los queman, provocando con ello un grave daño a la machi personalmente, pues con ello la machi sufre severos dolores físicos y daños espirituales. Por otro lado provoca una profunda y muy agrietada relación intracomunidad, e intrafamiliar.

Una de las principales razones de estos juicios y acciones provienen del alejamiento de nuestros hermanos de sus conocimientos, del mapunche kimün. Al mismo tiempo de ir adoptando un conocimiento wigka de diversos aspectos, pero muchas veces parcial, alimentado por intenciones dogmáticas.

Otro aspecto que coarta la posibildad de la concreción del machin es el económico. Los ceremoniales para el machilün y el anümrewen, significan un alto costo, pues ellos duran varios días, requieren de la participación de muchas personas, del lof entero y el reyñma. Además el trabajo de iniciar a un machi por otro, es de alto costo, pues implica también un alto riesgo para ambos y es un acto de importancia trascendental.

\section{Algunas razones por las que es aceptado el rol de machi en la familia}

Machi ta yamgekey. La machi es una persona respetada, de prestigio en la sociedad mapuche, por su capacidad, su desempeño, sus conocimientos y dedicación a su oficio.

Küme machigele waj mapu rupayay. Un machi generalmente sale de su comunidad hacia otras latitudes en donde se le ha escuhado que es una küme machi, buena machi. A mayor reconocimiento, mayor requerimiento.

\section{¿CHUMGECHI MACHIGEKEY CHE? \\ ¿COMO SE LLEGA A SER MACHI?}

El fenómeno que lo causa es el perimontun, visión que la persona tiene del geh que le hace el llamado a asumir el rol. Este fenómeno acontece a muy temprana edad en las personas llamadas a ser machi. También se revela a través del pewmatuwün, desde el mundo onírico. Pero cuando la persona no asume tal rol y lo evade, comienza a padecer los desiquilibrios de por vida. La cultura señala que mientras más joven se asuma el rol será más pertinente.

El origen de la machi es la naturaleza. Pues los newen que existen en ella, en el naüq mapu, son los que «toman» a los machi. Es una de las razones por la cual el mapuche le da tanta importancia a la naturaleza, pues en cada una de las manifestaciones del mapu, existe un newen que sustenta esa manifestación; menoko, cursos de aguas que mantienen anegado terrenos y bosques en gran parte del año; xayenko, cascadas y vertientes; wixunko, esteros; mawizantu, bosques montañosos; wigkul, cerros; wüfko, vertientes; xalkan, trueno, por nombrar algunos.

«Iñche kuyfi machigen, pichi zomogen machigen, fij püle rupan. Iñche ta fewla ta ñi machigenun, füxa kuyfi ta machigen zewma. Pichi malengen ta tuenew ta perimontun, Fücha chaw ta femkumuenew. Fücha Chaw ta elkunuenew, femgechi ta gijatuaymi, lukutuaymi, pürampayafiymi ta kom gijatun, kom lukutun, pipiyenew ta ñi wenu mapu Chaw». "Toro perimontungen iñche, ragi pichi foyentu perumefiñ kiñe pichi toro buyriffkülelu, fey ñi nüetew ti newen...» Caniullán.

Yo me hice machi desde muy joven, era solo una niña cuando me hice machi, pasé por muchos lugares. Mi oficio no es reciente, desde ya hace m, ucho tiempo que lo soy. Era solo una niña cuando tuve mi perimontun, Fücha Chaw me ha destinado para ello. Fücha Chaw me ha creado de esta forma, .de esta forma debes de realizar tus oraciones, de esta forma debes de arrodillarte, levantarás muchos gijatun. Eso es lo que me decía insistentemente mi creador. Mi visión se manifestó a través de un toro, en medio de un follaje del canelo, en el mallin, se me apareció un hermoso toro, fue la forma en que el gran poder se apoderó de mi. 
Elpperimontun se manifiesta de diferentes formas, y adopta diferentes seres de la naturaleza, ya sean reales e incluso seres no visibles.

Luego comienza el machi kuxan: enfermedad de machi, kisu kuxan: su propia enfermedad. Con estos términos se entienden las primeras manifestaciones de la posibilidad de que la persona sea machi. Estas manifestaciones van acompañadas de distintas dolencias que afectan física y sicológicamente a la persona.

El pewma en estos períodos será frecuente, altamente significativo y muy relacionado con el oficio. Elugeken kulxun ñi pewma, küymiken ñi pewma, umawtupuken ragi foyentu ñi pewma, pikey ñi güxam ti pu machigelu. Me pasaban un kulxug en mis sueños, luego comenzaba a bailar y entonces entraba al küymin, todo ello en medio del canelo. Son los sueños que suelen contar las personas que ahora son machi.

«Entonces todos sus amigos le dijeron: «Tiene que hacerse machi no más. Esa es una chiquilla todavía; si fuera mujer vieja, entonces se podría hablar de brujería; pero esa niñita no sabe nada de brujos. Seguro que el celestial creador de la gente la ha elegido para que sea una verdadera machi», Coña (1995).

Küpan mew machi püjügerkey tüfa, ñi epun chiken kukuyem fibewgekerkefulu am.

La persona que por ascendencia ha tenido que asumir un rol estructurado por sus antepasados. El espíritu se manifiesta en la persona elegida, independiente del género.

\section{¿CHEM CHUMKEY TA PU MACHI? \\ ¿CUAL ES LA FUNCIÓN Y EL ROL DE MACH?}

«La función de machi no es aspiración propia de una persona, sino que es una vocación divina. Es una persona seleccionada, escogida y predestimada por Dios. No es una carrera para ganar dinero, como ambición personal, sino que es una misión muy elevada que tiene que cumplir en esta tierra, haciendo el máximo bien entre sus semejantes...», Alonqueo (1979).

A nuestra primera vista, dos son las principales funciones de la machi. a) la de la medicina

b) presidir ceremonias religiosas.

En el primer rol y el más conocido, la machi despliega todo su conocimiento para tratar a su paciente. Pero dentro del tratamiento se incorpora el gübam el consejo tanto a su paciente como a su familia e incluso a su lof.

Otro de los roles importantes de los machi en los lof, se refiere a la de guiar algunas ceremonias de gran relevancia para el mundo mapuche. En el gijatun de sectores, bafkehche, naüq che y parte de los wenteche, la machi juega un rol fundamental, como lo hacen los gempiñ en el sector wijiche y los logko en gran parte del sector wenteche.

Una vez asumido el rol de machi, comienza a desarrollar su trabajo en donde la requieran. Ella deberá estar siempre disponible y presta a servir a sus demandantes. Los métodos de trabajo son variados, pewütun, pelotun, ulutun, zatun o machitun. Haciendo uso de su kimün, acerca de la herbolaria, preparará los baweh que genralmente son para beber, según la prescripción que ellos indican. "La buena machi debe conocer todo el material extraído del bosque, las yerbas nocivas y las medicinales, cortadas con diversas precauciones, $y$ otras sustancias de virtudes terapéuticas reconocidas», Guevara. (1912).

Este último autor recoge otro aspecto fundamental de la filosofia mapunche, que habla de la forma de relación en este caso del machi con respecto a la herbolaria. Las precaucíones con que se debe recoger las plantas medicinales, con el debido respeto y previa comunicación y autorización del geh del lugar.

«Las machis son distintas a las otras mujeres porque, según la creencia mapuche, son escogidas o llamadas sobre-naturalmente para ejercer su vocación mediante enfermedades inexplicables, sueños y visiones. Después de iniciadas están comprometidas con su profesión de por vida», Bacigalupo (1994).

La machi busca el origen de la enfermedad, el origen de la dolencia y no ataca la dolencia en sí. Ayuda a que el newen causante del mal retorne a sus oríge- 
nes, reestablece el equilibrio de la persona. En este tema aflora otra necesidad como lo es el origen de la enfermedad según la perspectiva mapuche, la que tendrá que ser abarcada en otra oportunidad.

\section{MACHIMOGEN \\ VIDADEMACHI}

«La vida de una machi es bastante difícil y acatrea una serie de responsabilidades que exigen el sacrificio de una serie de responsabilidades que exigen el sacrificio de una vida familiar normal. A cambio, las machis son personas prósperas y prestigiosas que obtienen respeto, autoridad e independencia en la comunidad», Bacigalupo (1994).

Ante todo, su oficio. Nada lo puede impedir. Existe una consagración a su rol a toda prueba, así lo demuestran algunos testimonios.

"Chuchi mapu am ta rupalan, kom mapu gujatuken, chew mapu rupan, gijatuyawün. $\mathrm{Ka}$ chumkawrume wiñonentuchekelan, wiñonentuchekefuli katikayafenew ga ñi Chaw Dios piken. Fey mew machi pigey ga machi müley ga ñi wiñonentuchenoal, ta ñi küme güxamkapual chew rume ga puwle, küme gijatupualu, küme lukutualu, küme nentualu ga ñi kimün, fey mew ta ayigekey ta bawehgelu, pekan pipigey ta bawehgelu füta Chaw ta kuxankatuafeyu ta ti», Caniullán.

Por muchas tierras he pasado, $y$ en todas ella he realizado mi gijatun, por todas las tierras, y además nunca he rechazado a algún enfermo, por si yo hiciera eso, mi creador, mi Chaw Dios me habría de castigar. Es por ello que la machi desde que es machi no debe rechazar a un enfermo, debe de saber conversar bien, adonde llegue, ella debe realizar un buen Gijatun, debe de saber arrodillarse en la madre tierra, para expresar sus buenos conocimientos, por ello la machi ha de ser querida y respetada por la gente.

Sus vidas se rigen por principios que emanan de su oficio, de su institución. Ser consecuente con su oficio es el principal desafio.
«La fiesta de geykurewen se lleva a efecto para iniciar y perfeccionar las machis nuevas. También la celebran las machis instaladas cuando renuevan su rehue; sólo por esos motivos se celebra», Coña (1995).

En el geykurewen, se hace partícipe a todas las comunidades y amigos del machi. Es una ceremonia religiosa y social. Generalmente la machi o el machi maestro o ta ti machiletew, está presente. Es un rito obligado llegado el tiempo de efectuarlo.

Muchas veces la machi se enferma, entra en desiquilibrio, y es necesario el geykurewen, y se procede también al müxumtun del newen del machi. Pero también se enferma cuando en las ceremonias no se cumplen los roles y no están disponibles los elementos necesarios para que la ceremonia sea completa. Cuando el zugumachife no responde a las expectativas del püjü.

«Müxümtu likan: Es el rito especial que cura a la machi cuando ésta ha sido abandonada por los espíritus benignos. Esto puede ocurrir en caso de haber alguna falla o percance grave en el ritual; también cuando la machi tiene algún sufrimiento grave por muerte de algún pariente, o cuando ella misma está gravemente enferma», Alcamán y Araya (1997).

Muchas veces el geykurewen consiste también en cambiar el madero. Se cambia por otro nuevo.

«Waj püle yegepaken ta ti chaw, kiñeke mew af zuamkefun, welu chem piafun am, bali wüla ta $\tilde{n} i$ femïawal piken».

"Kuyfi rume weza kuxankawfun em, kiñe komant'ü kawejutukefun ñi puwal ti kuxan mew, apümtukukefun mawüh, wüxe pikelafun em, fey tüfa kushelu iñche, rume kuxanchewtun ta ti». Luisa Manquel.(4)

De todas partes me solicitan, a veces ello me causa desgano, pero qué puedo hacer, mi deber es andar en ello hasta que muera. 
Antes yo sufrí mucho, dias entero a caballo para poder llegar hasta los enfermos y atenderlos, días enteros en que a veces me llovía todo el camino, grandes frío he consumido en mi labor, pero ahora que estoy anciana, la enfermedad se ha apoderado de mi por todas esas andanzas.

"Chem che rume ta akule, ta mi kayñegeafuy rume, müley ta mi zayafel, ta mi bawehtuafiel, ka xipa mojfeñchegepejele rume, müley ta mi küzawtuafiel, fey mew je no am ta machikunueyu. Pigen ñi pewma mew». Caniullan.

Cualquier persona que llegare, aun que el sea tu enemigo, tienes el deber de atenderlo, acudirlo, aun que sea de otra sangre (no mapuche), tienes el deber de recurrirlo con tus conocimientos en la medicina, es por ello que yo te he hecho machi. Eso eran algunos de los mandatos que en sueño he recibido

\section{ALGUNOS PROBLEMAS}

La institución machi, posee muchas interpretaciones desde la otra sociedad y su cultura como podría ser esperado, pero también ha surgido el problema de la descalificación y la interpretación negativa desde los propios hermanos mapuche como ya lo hemos detallado, quienes permeados por una idea foránea que los lleva a rechazar lo propio han rechazado esta institucionalidad e incluso, en algunos casos, llegando a violentarla dentro de su lof.

Otro problema que se ha sucitado y es fundamento para muchos detractores es el tema económico. Decir que la persona se hace machi por un interés económico. Sobre todo sectores más proclives a la modernización, si bien es cierto no niegan la función o importancia de la machi en todas sus dimensiones, lo asocian a una actividad de mero enriquecimiento. Aunque tampoco podemos negar que este fenómeno en algunos machi existe, tiene su explicación en sus relaciones con el mundo no mapuche, en donde son solicitados y son retribuídos magníficamente, en lo económico. Ello también ha servido para que las propias machi duden de los orígenes de otras. El cómo es posible un comportamiento poco ético de sus pares. Existen machi que no aceptan una retribución más allá de lo que ellos solicitan. No se debe exceder en el cobro, existen reglas para ello.

"Aunque los machis son prósperos económicamente, nadie puede escoger ser machi por conveniencia económica ya que son "elegidos sobre-naturalmente». Los machis deben tener fe en sí mismos y en sus poderes para curar pacientes», Bacigalupo (1994).

Ya hemos visto la forma de llegar a ser machi, y además podemos agregar, que prácticamente ninguna machi, ha querido en un principio aceptar tal rol, por sus complejas significaciones y sufrimiento que ello significa por un lado y la abnegación y entrega por otro.

\section{BIBLIOGRAFIA}

ALCAMAN, S. y J. ARAYA (1997). Manifestaciones Culturales Y Religiosas del Pueblo Mapuche. Ed. Fundación Instituto Indígena, Temuco, Chile.

ALONQUEO, M. (1979). Instituciones Religiosas del Pueblo Mapuche. Ed. Nueva Universidad. Pontificia Universidad Católica de Chile.

BACIGALUPO, A. M. (1994). El Poder de las Machis Mujeres en losValles centrales de la Araucanía. En Comprensión del Pensamiento Indígena a Través de sus Expresiones Verbales.

COÑA, P. (1995). Lonco Pascual Coña ñi Tuculpazugun Ed. Pehuen,Santiago Chile.

DURAN, T., J. QUIDEL, E. HAUENSTEIN, L. HUAIQUIMIL, Z. INOSTROZA, E. PICHUN, F. CAÑUlaF, O. ALCHAO, L. ANTINAO, S. CANIULEMPI Y M. PICHUN (1997). Conocimientos y Vivencias de Dos Familias Wenteche Sobre Medicina Mapuche. Ed. LOM, Santiago, Chile.

GUEVARA, T. (1912). Ultimas Familias y Costumbres Araucanas. Ed. Barcelona, Santiago, Chile. 